\title{
Mechanisms and applications of in-situ excess sludge reduction strategies: a review*
}

\author{
Wan-Qian Guo, Xiao-Dong Wang and Qing-Lian Wu \\ State Key Laboratory of Urban Water Resource and Environment, \\ Harbin Institute of Technology, \\ Harbin 150090, PR China. \\ E-mail: guowanqian@126.com
}

\begin{abstract}
Nowadays, with the constant development of wastewater treatment processes, more and more waste activated sludge has been produced which cannot be overlooked. To solve this problem, some strategies have been employed in-situ to reduce waste activated sludge production and proved effective. This paper reviews mechanisms and applications of the in-situ excess sludge reduction strategies. It is expected that this paper could attract widely attention on sludge reduction and inspire researchers and engineers to develop feasible and effective measures to control sludge production in an acceptable range.
\end{abstract}

Keywords: Sludge Reduction; In-Situ; Lysis-Cryptic Growth; Uncoupling Metabolism; Worms' Predation.

\section{Introduction}

In recent years, conventional activated sludge (CAS) process is the most widely used for both municipal and industrial wastewaters. One of the drawbacks of the CAS process is, however, its high sludge production, in which way biomass yield coefficient in this process was approximately $0.5 \mathrm{~kg}$ of biomass per removal of $1 \mathrm{~kg}$ of COD removed [1].

According to statistics, the USA currently generates about 8.2 million tons of dry WAS per year, and the EU annually produces over 10 million tons. In China, the total amount of dry sludge is about 4.4 million tons in 2010 (ChinaWater.net, 2011) and is estimated to increase constantly in followed few years .

Nowadays, some strategies have been employed in-situ to reduce waste activated sludge production and proved to be effective. It is usually divided by mechanism into four parts: Lysis-cryptic growth, Uncoupling metabolism,

\footnotetext{
* This work was financially supported by young top-notch talent teachers by Harbin Institute of Technology (AUGA5710052514). The authors also gratefully acknowledge the financial support by State Key Laboratory of Urban Water Resource and Environment (2014TS06).
} 
Predation on bacteria and Maintenance metabolism [1]. This paper reviews mechanisms and applications of these in-situ excess sludge reduction strategies.

\section{Lysis-Cryptic Growth}

Sludge reduction by lysis-cryptic growth has been studied for a long time and applied successfully to some extent. The mechanism has two aspects: microbe cells lysis and cryptic growth. In some certain conditions, membranes dissolve and cells are lysed first. When a cell is lysed, contents and nutrients stored in it are released in the sewage environment. The organic materials released from the cell lysis increase the organic load of wastewater, and, as an autochthonous substrate, enter in the metabolism cycle of other microorganisms.

The biomass growth on organic lysate is different from growth on original substrate because it is not present from the beginning, while cannot be differentiated separately from the growth on original substrate, and therefore termed as cryptic growth [2].

As reported, there are many ways to achieve lysis-cryptic growth such as chemical oxidation, ultrasound treatment and some other methods. Nie et al.[3] showed a zero yield of excess sludge was achieved at an ozone dose of $100 \mathrm{mg}$ $\mathrm{O}_{3} \mathrm{~g}^{-1} \mathrm{SS}$ in the sludge-reduction system during a continuous-operating period of 45 days. Li et al.[4] studied waste activated sludge reduction using sludge sonication and showed that the amount of sludge could be reduced by $58.8 \%$ when ultrasonic intensity was $1.6 \mathrm{~W} / \mathrm{ml}$ and the sonication time $15 \mathrm{~min}$. Toshikazu et al.[5] applied a high-pressure jet device (HPJD) as an alternative technology to solubilize sludge and showed that the degree of reduction was $12.2 \%$ and the viable microorganisms decreased by $42.8 \%$.

\section{Uncoupling Metabolism}

Under normal conditions, most energy produced in catabolism is used to maintain microbial activities and anabolism which produces more biomass so that catabolism and anabolism are well coupled. While in some certain conditions, catabolism no longer couples to anabolism so that most energy is wasted and little biomass is generated, and therefore termed as uncoupling metabolism.

Till now, many conditions of uncoupled metabolism have been studied out such as the presence of uncouplers, heavy metals, abnormal temperatures, limitation of nutrients and excess energy source. Chen et al.[6] reported $40.0 \%$ of the excess sludge yield was reduced by adding $0.8-1.0 \mathrm{mg} / \mathrm{L}$ TCS. Feng et al.[7] exploited a composited uncoupler consisting of TCS at $0.8 \mathrm{mg} / \mathrm{L}$ and TCP at $2.8 \mathrm{mg} / \mathrm{L}$, and the observed growth yield (Yobs) was reduced from 0.72 to 
0.398. Besides, the follow-up work found the adding of composited unpoupler has no negative effects on sewage treatment efficiency.

\section{Predation on Bacteria}

Biological treatment process of wastewater and the environment for this treatment is just like a man-made ecosystem in which various microorganisms live as activated sludge. When the microfauna preyed on the microorganisms, only 1/10 energy is transferred into the next nutrition level, and the predator in the conversion process consumed 9/10 energy [8]. Most energy is consumed so that biological solid production decreases i.e. sludge reduction.

Two-stage sludge predation system, to control the application and distribution of predators, is a pattern that the first stage is referred to the bacterial stage without biomass retention; and the second stage is designed as a predator stage (activated sludge system) with a long SRT for the growth of protozoa and metazoan [9]. It is reported about $60-80 \%$ sludge yield was reduced in the second stage compared with that in the first stage.

\section{Maintenance Metabolism}

In all species of microorganisms, the energy obtained and captured in the form of ATP during biological oxidation is used by the microbial cells for their maintenance followed by synthesis. That is, when the energy is lacking, microbial cells use it for vital activities most and for synthesis rarely. Such metabolism in which the energy provided by substrate is spent most on maintenance of cell vital activities rather than new biomass synthesis is termed as maintenance metabolism.

Up to now, two ways are studied most to achieve this metabolism which are reduction of sludge discharge and high sludge retention time respectively. And the main method to achieve maintenance metabolism is to extend sludge retention time and the corresponding most typical technology is MBR process at present. It is reported that MBR process strengthens maintenance metabolism and reduces excess sludge production and can achieve no excess sludge production even [10].

\section{Conclusion}

Compared with follow-up sludge reduction, the in-situ excess sludge reduction strategies are more economic, more effective and more promising. Of course, further study is needed to perfect these strategies. For example, we can study the combined effects of each other like the composited uncoupler consisting of TCS 
and TCP. Besides, the combination of different mechanisms may be effective and practical, and worth further study.

\section{References}

1. Ahmad Reza Mohammadi, Nasser Mehrdadi, Gholamreza Nabi Bidhendi, Ali Torabian. Excess sludge reduction using ultrasonic waves in biological wastewater treatment. Desalination 2011;275: 67-73.

2. Anwar Khursheed, A.A. Kazmi. Retrospective of ecological approaches to excess sludge reduction. Water research 2011;45: 4287-4310.

3. Yafeng Nie, Zhimin Qiang, Weiwei Ben, Junxin Liu. Removal of endocrine-disrupting chemicals and conventional pollutants in a continuousoperating activated sludge process integrated with ozonation for excess sludge reduction. Chemosphere 2014;105: 133-138.

4. W. Li, G. Zhang, P. Zhang, H. Liu, International Journal of Biotechnology 2008;10: 64 .

5. Toshikazu Suenaga, Mio Nishimura et al. High-pressure jet device for activated sludge reduction: Feasibility of sludge solubilization. Biochemical Engineering Journal 2015;100: 1-8.

6. Chen GH, Mo HK, Liu Yu. Utilization of a metabolic uncoupler, 3,3', $4^{\prime}, 5-$ tetrachlorosalicylanilide (TCS) to reduce sludge growth in activated sludge culture. Water Res 2002;36: 2077-2083.

7. Feng Xiaochi. The characteristics and effects of a reducing sludge composited uncoupler on treatment efficiency. (in chinese) 2013.

8. Wan-Qian Guo, Shan-Shan Yang, Wen-Sheng Xiang, Xiang-Jing Wang, Nan-Qi Ren. Minimization of excess sludge production by in-situ activated sludge treatment processes-A comprehensive review. Biotechnology Advances 2013;31: 1386-1396.

9. Ratsak CH. Grazer induced sludge reduction in wastewater treatment. the Netherlands: Vrije Universiteit; 1994 [PhD thesis] .

10. Yoon, S.-H., Kim, H.-S., Yeom, I.-T. The optimum operational condition of membrane bioreactor (MBR): cost estimation of aeration and sludge treatment. Water Research 2004; 38: 37-46 . 